\title{
DESIGN AND PROTOTYPING OF A MEMS-BASED CRACKMETER FOR STRUCTURAL MONITORING
}

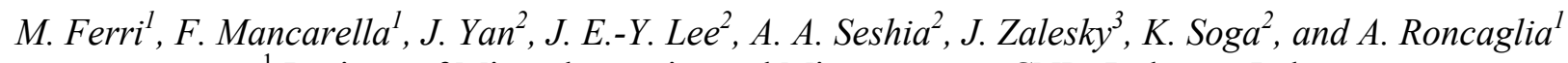 \\ ${ }^{1}$ Institute of Microelectronics and Microsystems, CNR, Bologna, Italy \\ ${ }^{2}$ Department of Engineering, University of Cambridge, Cambridge, United Kingdom \\ ${ }^{3}$ Czech Technical University in Prague, Faculty of Civil Engineering, Prague, Czech Republic
}

\begin{abstract}
Crack measurement is an important technique in structural monitoring, which is presently implemented with macroscopic devices characterized by rather demanding power consumption requirements. Such devices, consequently, are not very well suited for wireless operation, which is particularly interesting for specific applications involving deployment of a number of crackmeters within large-scale ageing infrastructures with possibility of remote, on-demand sensor interrogation. This paper reports about the research work related to the design and prototyping of a novel crackmeter suited for wireless structural monitoring realized with silicon MEMS strain sensors with high resolution, very low power operation and small size.
\end{abstract}

\section{KEYWORDS}

Crack monitoring, wireless, strain sensors, MEMS

\section{INTRODUCTION}

Crack measurement is a widespread technique in structural monitoring, since the movement of existing cracks is often an alarming indicator of possible instabilities in civil infrastructures. The best crackmeters presently available on the market are based on the vibrating wire principle [1]. These sensors can be mounted across a wall crack and monitor its movement through the shift in their vibration frequency, due to applied strain. Since their power consumption is rather high (tens of $\mathrm{mW}$ ) these sensors are not very well suited for wireless operation, which would be particularly interesting for specific applications involving deployment of a number of crackmeters within large-scale ageing infrastructures like underground tunnels, with possibility of remote, on-demand sensor interrogation.

Through the use of silicon MEMS technology, strain sensors with high resolution, low power operation and small size can be developed [2]. In this paper the research work related to the design and prototyping of a novel crackmeter suited for wireless structural monitoring realized with MEMS strain sensors is reported.

\section{DESIGN}

\section{Crackmeter Design}

The crackmeter is realized with a thin steel bar fixed across the crack on the tunnel wall, onto which a multi-directional MEMS strain sensor is soldered (Fig. 1).
A movement of the wall crack (contraction or expansion) can be detected by the sensors through the strain generated on the steel bar and transferred to the silicon chip. Since the resonant MEMS sensors are operated through a self-sustained electrostatic actuation using an electronic oscillation loop, no static bias current is needed for their operation and the dynamic power requirements can be comparatively small. These features, together with their high strain sensitivity, make these devices attractive for crackmeters.

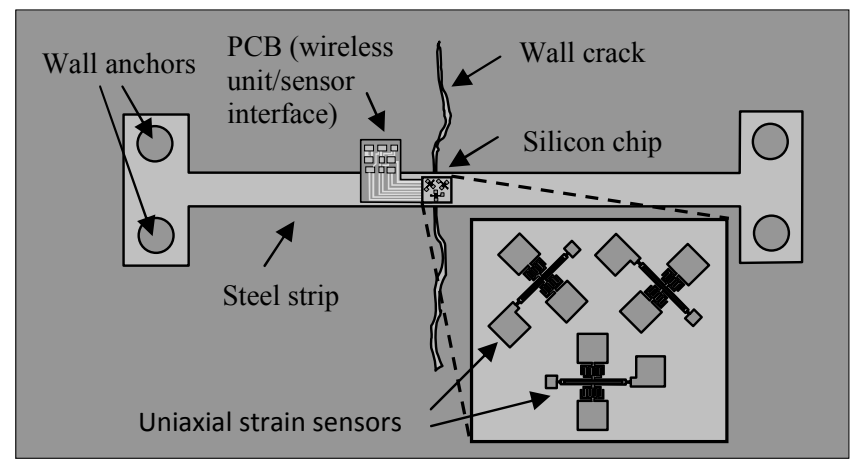

Figure 1: Concept of MEMS-based crackmeter operating in wireless mode.
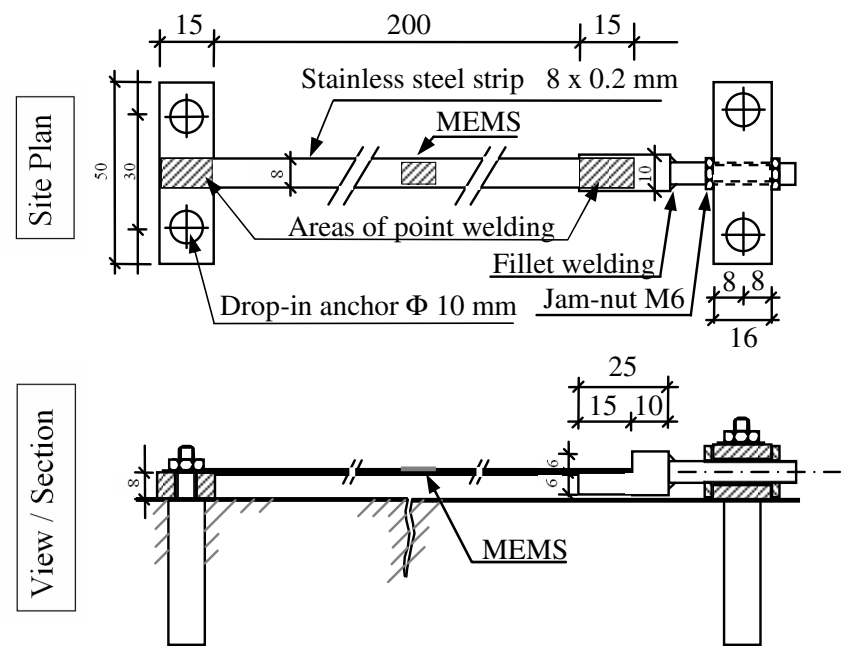

Figure 2: Crackmeter mechanical design: top view (top) and cross-section (bottom), dimensions in $\mathrm{mm}$.

The mechanical design of the crackmeter is illustrated 
in Fig. 2. It is composed by a $200 \mathrm{~mm}$ long steel strip connected by point welding to anchors suited for mounting the device across a crack on a concrete wall. The strip thickness is $0.2 \mathrm{~mm}$ and the width is $8 \mathrm{~mm}$. This geometry has been conceived in order to pre-stress the support steel strip to measure possible crack contractions or extensions in the range $(0.01-0.1 \mathrm{~mm})$, with no significant tensile force applied on the crack, considering a maximum crackmeter extension of $0.05 \%$. The steel strip deformations induced by the crack movements are detected by a silicon MEMS strain sensor based on mechanical resonance frequency shift, which is soldered on the strip with effective strain coupling from steel to silicon and packaged in vacuum (see Section "Crackmeter Prototyping").

\section{MEMS Strain Sensor Design}

The resonant strain sensor used in the crackmeter is a novel $\mathrm{H}$-framework flexural mode resonator, which utilizes a central beam to mechanically couple twin clamped-clamped beam resonators into vibration in the second harmonic mode as illustrated in Fig. 3.

The resonator is grounded and electrostatically driven into vibration by two interconnected electrodes and capacitive sensing of the induced displacement is through a pair of similar interconnected electrodes as shown in Fig. 3. Through this electrode arrangement, vibrations in the preferential mode are excited and modes that result in an out-of-phase capacitance variation between the driving / sensing electrodes and the resonator itself are suppressed.

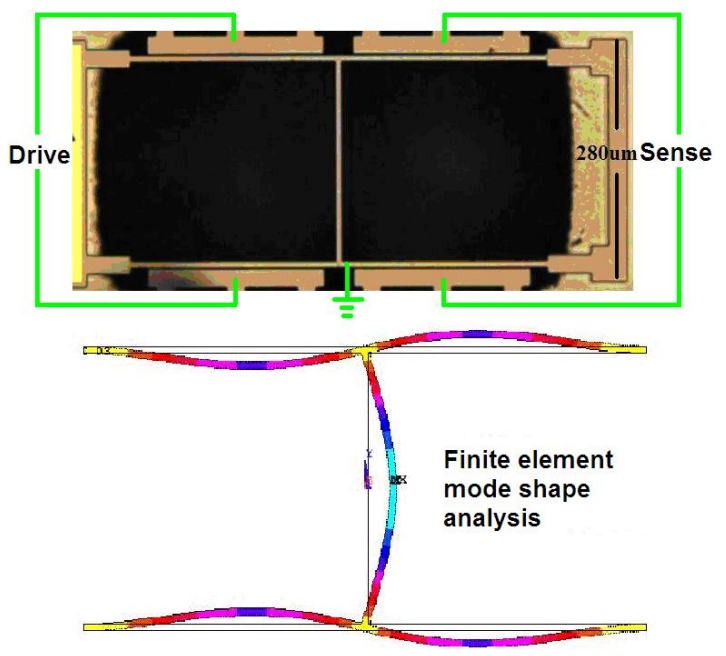

Figure 3: Optical micrograph and FEA simulation of the silicon MEMS strain sensor used in the crackmeter.

\section{EXPERIMENTS}

\section{Crackmeter Prototyping}

In order to make the steel strip connection to the anchors as stiff as possible, arc point welding was employed
(Fig. 4). The material used for the steel strip and the anchor was stainless steel Aisi 304.

The steel strip was cut from a $50 \times 50 \mathrm{~cm}^{2}, 0.1 \mathrm{~mm}$ thick commercial steel stainless steel Aisi 304 foil using a mechanical cutting tool, according to the geometry reported in Fig. 2. The anchors were grinded on the same material starting from a bar with a $16 \times 16 \mathrm{~mm}^{2}$ square section. The steel strip was equipped by a nut which allows for changing the unstrained length of the crackmeter.

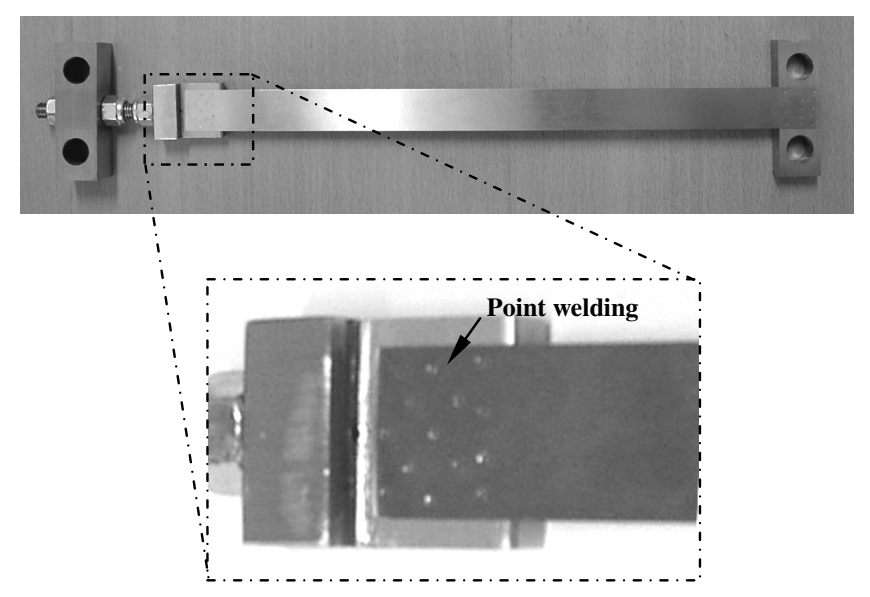

Figure 4: MEMS-based crackmeter prototype (steel parts).

For the silicon chip bonding and vacuum packaging on steel, techniques previously developed by our group and described in [3] have been adopted (Fig. 5).

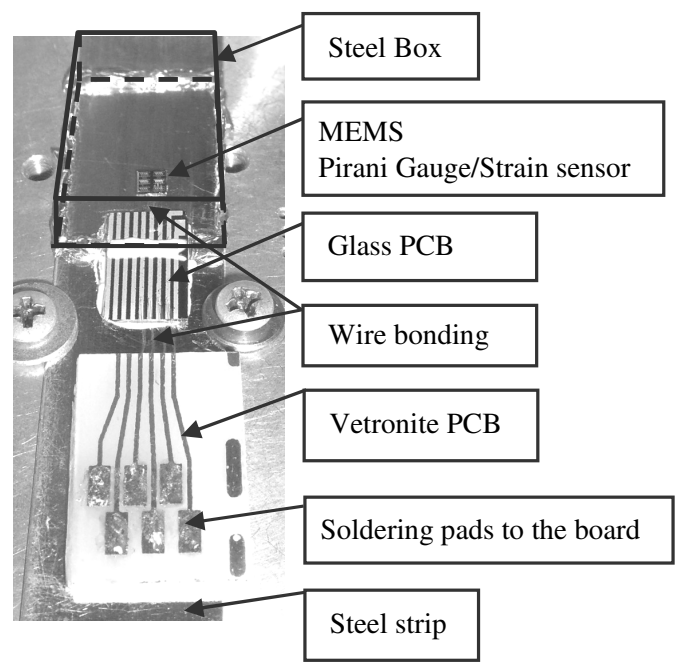

Figure 5: Packaging scheme used in the MEMS crackmeter.

In order to ensure an optimal performance of the MEMS strain sensor, experiments aimed at achieving a vacuum packaging of the silicon device when bonded to the 
steel bar have been carried out.

\section{MEMS Vacuum Packaging}

For the vacuum packaging, two methods were tested. Both were based on the adhesive bonding of a steel box on the crackmeter bar, performed using vacuum glue (Torr Seal from Varian).

In the first solution, vacuum was created inside the glued steel box using a rotary pump connected by means of a glass pipe, also fixed to the chamber inlet by vacuum glue (Fig. 6). After the required vacuum level was reached (pressure around $10^{-3}$ Torr), a methane flame was used to heat the glass pipe up to its softening temperature. The heated glass was then pressed with pliers and sealed, also exploiting the collapse effect induced by the vacuum inside. The glass pipe was finally cut while still hot so that, after cooling, the pressed part of the pipe was soldered to vacuum grade.
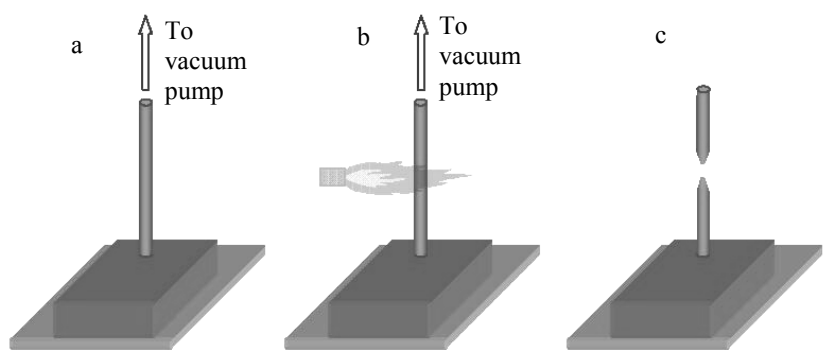

Figure 6: Step sequence used to obtain a sealed vacuum packaging with the glass pipe method.

In Fig. 7, a crackmeter prototype implemented with the glass pipe vacuum method is reported.

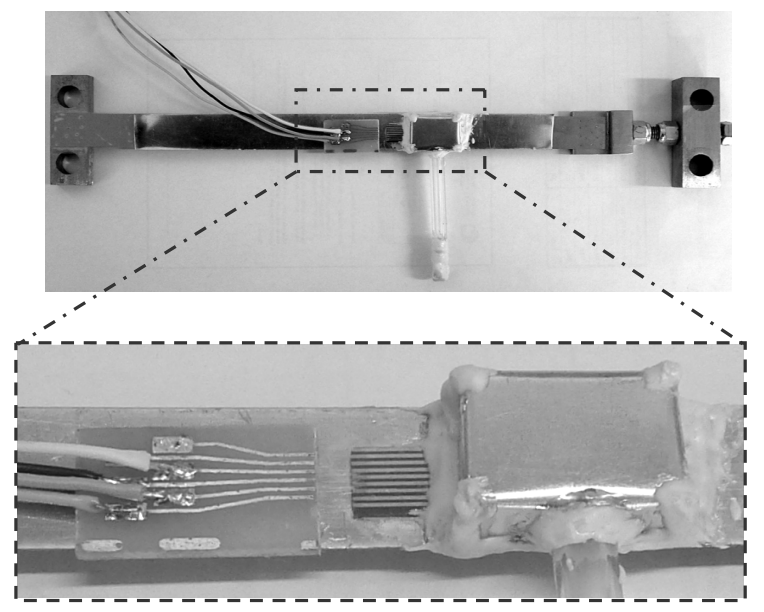

Figure 7: Crackmeter prototype with glass-pipe vacuum-sealed package.

With the second technique, a similar evacuation procedure was implemented, but instead of the glass pipe a tin-lead tube was used as an inlet for the chamber (Fig. 8). The tin-lead tube was temporary connected to a glass pipe in order to ease its connection with the pump. Once reached the desired vacuum level, the glass was heated up to roughly $200{ }^{\circ} \mathrm{C}$ in order to melt the tin tube inside. As a consequence of tin-lead melting, the metal tube collapsed on the chamber inlet, creating a vacuum tight sealing. The temporary glass pipe can be removed after cooling down to room temperature.
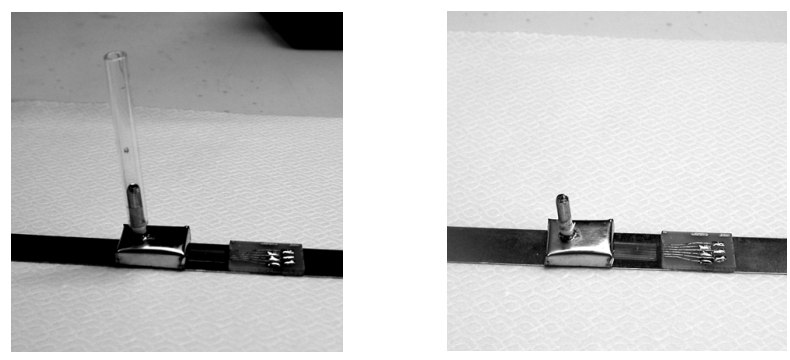

Figure 8: Crackmeter prototype detail with metal soldering vacuum-sealed package before (left) and after (right) glass removal.

Several experiments were performed with both methods, and the performance evaluated by monitoring the vacuum level created inside the chamber for several days after sealing (a micro-Pirani gauge inserted into the chamber was used for these measurements). The best results obtained with the two different methods are reported in Fig. 9.
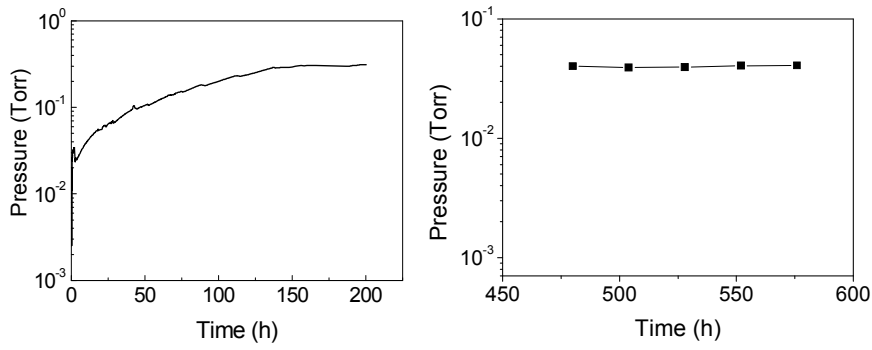

Figure 9. Chamber pressure evolution after sealing using the glass pipe (left) and metal soldering (right) method.

As may be observed, the metal soldering method permitted to achieve a higher vacuum level than the glass pipe one (below $10^{-1}$ Torr). Moreover, this method also demonstrated a higher reliability, since the glass softening and sealing operation when using the pipe often gave rise to failures due to micro-cracks formed within the glass.

\section{Silicon/steel mechanical coupling}

In order to bond the silicon chip to steel with effective strain transfer, a novel soldering method based on $\mathrm{Cu}-\mathrm{Sn}$ 
eutectic alloying was applied (Fig. 10).

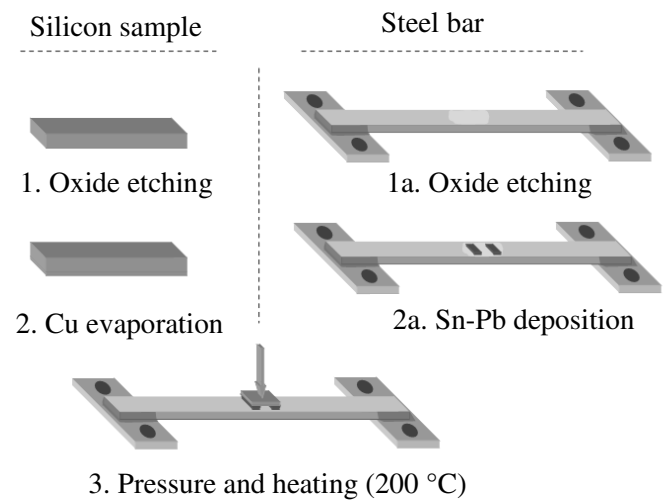

Figure 10: Soldering method employed to fix the MEMS strain sensor to the steel bar of the crackmeter.

After cleaning the samples with isopropanol and acetone, the native oxide layers were removed by vapor phase $\mathrm{HF}$ etching at $40^{\circ} \mathrm{C}$ on silicon and using a standard de-oxidant agent on steel. Afterwards, a roughly $500 \mathrm{~nm}$ thick copper layer was deposited by evaporation on the backside of the silicon chip, while a tin-lead alloy spot was defined on the steel surface by thermal welding. In order to achieve eutectic bonding, the side of the chip covered with copper was placed in contact with the tin welding on steel and heated at $200{ }^{\circ} \mathrm{C}$ for $10 \mathrm{sec}$ under applied pressure. The strain transfer from steel to the soldered silicon chip was evaluated with the aid of commercial strain sensors (from Vishay), yielding a value around $95 \%$ (Fig. 11).

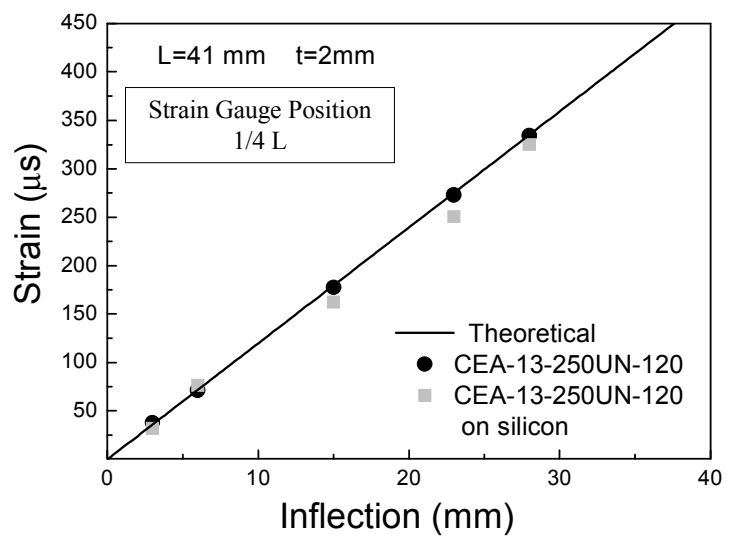

Figure 11: Steel/soldered silicon strain transfer test results

\section{MEMS strain sensor testing}

Initial open-loop measurements on the MEMS-based crackmeter prototype are reported in Fig. 12. In these measurements, the open-loop device transmission is plotted as a function of electrostatically induced applied axial strain. The results show that a 0.079 micro-strain results in a frequency shift of $16 \mathrm{~Hz}$. The measurements contrast with a minimum detectable frequency shift of $3.1 \mathrm{mHz}$ previously demonstrated for a closed-loop double-ended tuning fork sensor fabricated in our group [4] allowing for strain resolution potential below $100 \mathrm{p} \varepsilon$ for a $1 \mathrm{~s}$ averaging time.

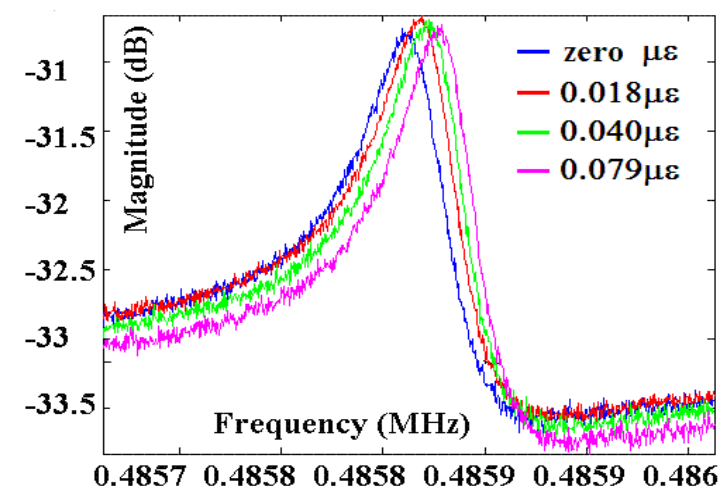

Figure 12: Open-loop measurements demonstrating functionality of the MEMS strain sensor through electrostatically induced micro strain.

\section{CONCLUSIONS}

The design and prototyping of a novel MEMS-based crackmeter for low-power, wireless structural monitoring has been described. Vacuum packaging of MEMS strain sensors soldered on a steel bar has been implemented in the crackmeter prototype, with an achievable vacuum level below $10^{-1}$ Torr and a strain transfer from silicon to steel around $95 \%$. A novel $\mathrm{H}$-shaped resonator has been used as a strain sensor, providing a peak frequency shift of $16 \mathrm{~Hz}$ for an electrostatically-induced solicitation of 0.079 micro strain.

\section{ACKNOWLEDGEMENTS}

This work, as a part of the European Science Foundation EUROCORES program S3T, was supported by funds from the ESF English, Spanish, Italian and Czech funding agencies and the EC 6th Framework Program.

\section{REFERENCES}

[1] http://www.geokon.com

[2] A. P. Pisano, "MEMS and Nanotechnology for the Handheld, Portable Electronic and the Automotive Markets", in Digest Tech. Papers Transducers'07 Conference, Lyon, June 10-14, 2007, pp. 1-4.

[3] M. Ferri, S. Cristiani, Y. Kobayashi, K. Soga and A. Roncaglia, "A packaging technique for silicon MEMS strain sensors on steel", Proceedings of IEEE Sensors 2008, Lecce, Oct. 26-29, pp. 1524-1527.

[4] J. E-Y. Lee, B. Bahreyni and A. A. Seshia, "An axial strain modulated double-ended tuning fork electrometer", Sensors and Actuators, Part A: Physical, Vol. 148, No. 2, pp. 395-400, 2008.

\section{CONTACT}

* Dr. Alberto Roncaglia, tel: +39-051-6399122; roncaglia@bo.imm.cnr.it 